\title{
Negative Effects of Industrial Clustering on Region Social and Economic Development: System and Agglomeration Approach
}

\author{
Dmitry Koshcheev ${ }^{1, *}$, Elena Tretiakova ${ }^{1}$, and Loan Dao Thi Sgoc $^{2}$ \\ ${ }^{1}$ HSE University, 614060 Perm, Russia \\ ${ }^{2}$ Smile Up English Centre, 66700 Di Linh, Vietnam
}

\begin{abstract}
The Present investigation illustrates the negative side of industrial clustering which often makes regional social and economic policy ineffective. Analyzing data from 790 papers, we identified and described 5 key negative clustering effects. Drawing from a system and agglomeration approach we propose a conceptual mechanism that illustrates cluster influence on a region, focusing on internal parameters which determine the impact nature (positive, negative or neutral). The investigation showed that the genesis of all the negative effects is connected with the tendency of industrial clusters to close in on itself in the medium term. Based on these findings, we propose recommendations on industrial cluster negative influence minimization.
\end{abstract}

\section{Introduction}

Among many concepts and models of regional policy, cluster approach has been one of the most popular territory development instruments among regional policy makers since 1990. However, despite the approach's popularity and its widespread use, investigations published in 2012-2020 showed a mismatch between the theory of industrial clustering and empirical data on cluster influence upon the region [1]. Theoretically, this influence is often thought of as an unambiguously good thing, but, empirically, there are only a few world famous successful clustering initiatives (Silicon Valley, Tokyo, Northern Sydney etc). Meanwhile most other industrial clusters (based upon the same principles as the effective ones) have neutral or, even, negative effects on social and economic development of the regions where they are situated [2]. One of the main reasons for this is insufficient knowledge of industrial cluster and region interaction mechanism, which functioning is still unclear [3].

As a consequence, regional policymakers, who are responsible for industrial clustering, cannot identify potential negative effects of clustering and minimize them. Hence, successful cluster initiatives are still rare.

This paper presents some key results of the conceptual investigation which had two main purposes. The first purpose was to better systematize and structure the part of cluster

\footnotetext{
*Corresponding author: DAKoshcheev@hse.ru
} 
theory which illustrates the industrial cluster negative effects on social and economic development of a region. The second purpose was to form the conceptual model, illustrating the process and conditions of the emerging negative effects mentioned.

\section{Materials and Methods}

Theory systematization is a complex task, which adopts a relatively narrow range of theorizing methods. However, most of these methods are ill-suited to identify and describe multiple relations between cluster theory elements and other close theoretical spaces. As a consequence, modern attempts to systematize cluster theory, which are based on a classical approach to conceptual analysis form a breeding ground for criticism, concerning the disability of the approach mentioned to fulfill some key criteria of a good conceptual analysis, including: complexity, completeness, historicity and systematicity. Within the present investigation, to fulfill the criteria mentioned, we use our own «system and criterion approach» (Fig 1).

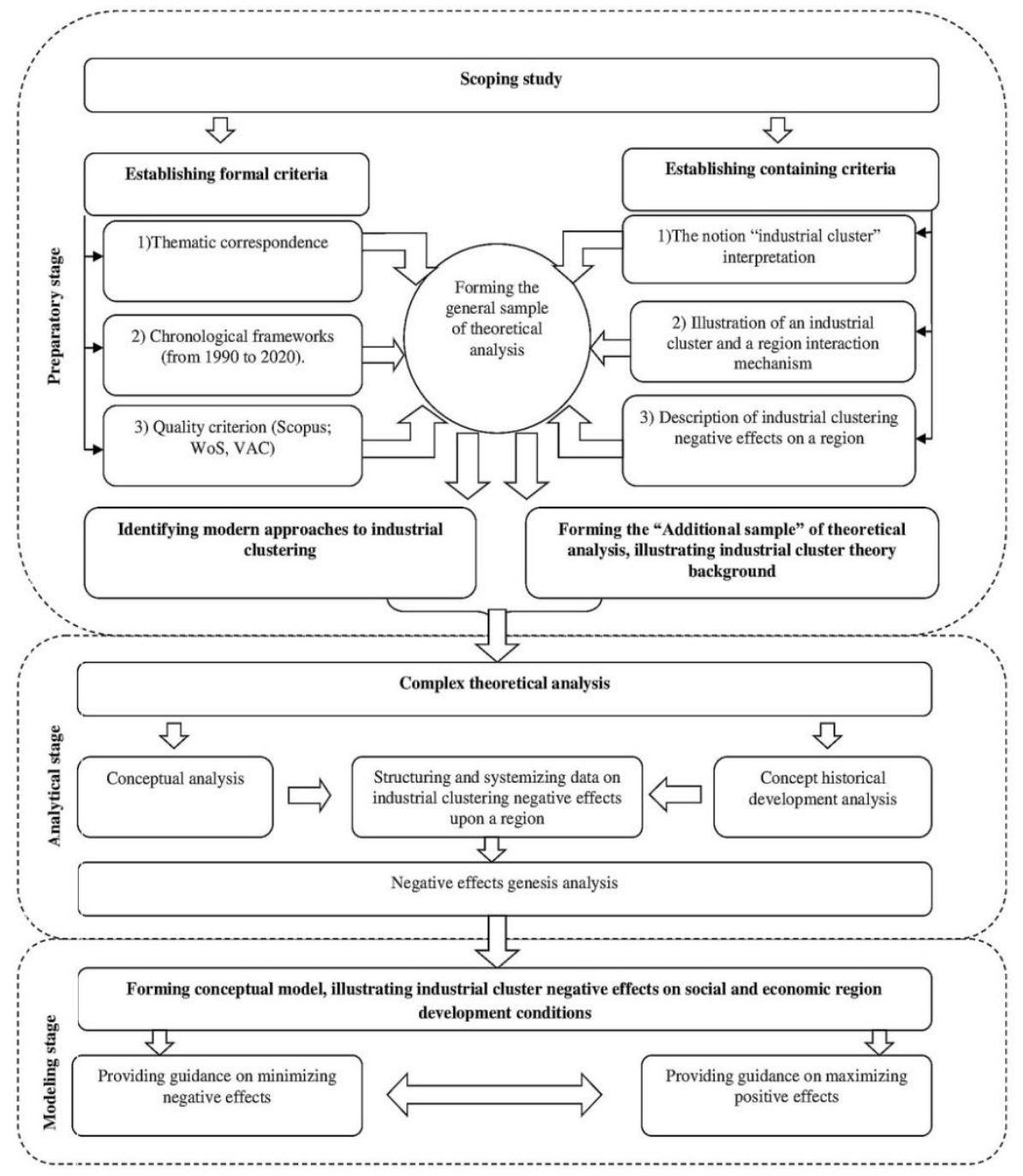

Fig. 1. System and criterion approach to theoretical analysis. 
The present approach focuses on three main stages of conceptual analysis, including preparatory stage, analytical stage and modeling stage.

The preparatory stage can be characterized by a search process of suitable publications and establishing two blocks of criteria: "Formal" and "Containing". "Formal" criteria illustrate external features of research papers, which satisfy the purpose of the investigation. "Containing" criteria reflect their internal features.Applying these sets of criteria for three key bibliometric bases (WoS, Scopus and Elibrary.ru), we form a general sample of theoretical analysis.

Drawing from the general sample, we use the a "step-back" method to form an additional sample, illustrating the background of cluster theory. The analytical stage focuses on conceptual approaches identification within cluster theory and their further description. On this stage, we analyze each approach two times. Firstly we consider the essence of each approach. Secondly, each approach is considered within its historical development. The analytical stage runs out as soon as views on negative effects of industrial clustering are identified within each theoretical approach. The modeling stage focuses on creating a conceptual model, which illustrates the mechanism and emergence conditions of industrial clustering negative effects on regional, social and economic development.

The mechanism mentioned sheds some light on how the negative effects of industrial clustering could be minimized, and, at the same time, how its positive influence could be maximized.

\section{Results and Discussion}

Drawing from the methodology mentioned, we have formed a general sample (704 papers) and an additional sample (86 papers).

Theoretical analysis showed that the complex vision of industrial cluster negative effects in spatial economics has been formed after the year 1990, when M.E. Porter suggested his new interpretation of the category "Industrial cluster" [4].

However, the conceptual analysis of the additional sample showed that the complex vision mentioned has its intellectual roots in some academic schools, formed within economic geography and spatial economics in the 19th and 20th centuries. For example, a classical school within "Third Italy studies" showed that clustering stimulates labor force spillovers from districts situated relatively far from the industrial cluster. As a consequence, it contributes to the rise of inequality in the social and economic development of living points and territories within one region [5]. Social and geographic schools, described the phenomenon of a firm's overconcentration in the territory of one cluster, which often decreases the rate of regional growth [6]. The institutional theory school showed the correlation between the increase of an industrial cluster efficiency and the rise of market entry barriers [7]. The Soviet economic theory school, considering industrial clusters as artificial units, showed that any potential mistake in their projecting may lead to a loss in cluster efficiency, which therefore will decrease the efficiency of regional budgeting [8]. The Mathematic and statistics school encapsulated some key principles of the industrial cluster influence on labour forces allocation between industries [9]. The nature of the influence mentioned is determined by regional social and economic circumstances: in some cases, this influence could be positive, while in others it is negative.

In 1990, the new approach to industrial clustering, proposed by M.E. Porter, formed a conceptual basis for modern cluster theory. At the early stage of remodified theory development, M.E. Porter described some potential "weaknesses" of industrial clustering, including its reduction effect clustered industries flexibility [4]. Meanwhile, the first complex attempt to describe the negative effects of industrial clustering is often associated 
with P. Krugman's «Geography and trade». This paper describes the interdependence between the growth rate of transport sector development and the level of clustering in a particular region.

Since then, the interest in the negative side of industrial clustering has grown unabated. However, the investigations on the topic mentioned were not realized in a systematic and complex way. As a consequence, the genesis of these effects is still unclear.

Drawing on concept operationalization, comparative analysis and theoretical study, we identified 5 negative effects of industrial clustering on regional, social and economic growth, which are shared by most of the researchers (Table 1).

Table 1. Font styles for a reference

\begin{tabular}{|c|c|c|}
\hline Effect & Interpretation \\
\hline Lock-in effect & $\begin{array}{c}\text { Potential focus of clustered firms on local contracts, which often leads to a lack } \\
\text { of flexibility and, therefore, disability to adapt to external market changes }\end{array}$ \\
\hline $\begin{array}{c}\text { Vulnerability to } \\
\text { specialization effect }\end{array}$ & $\begin{array}{c}\text { Clustered firms develop their specialization within a value-added chain, to } \\
\text { increase quality of the final product. However, in doing so, they decrease the } \\
\text { economic flexibility of the whole region industrial system }\end{array}$ \\
\hline $\begin{array}{c}\text { Overconcentration } \\
\text { effect }\end{array}$ & $\begin{array}{c}\text { Clustering attracts small and medium size firms to the living points, associated } \\
\text { with an industrial cluster. This process increases competition within the cluster } \\
\text { area. A high rate of competition may decrease the ability of small and medium } \\
\text { size firms to survive. This tendency often results in negative dynamics of region } \\
\text { social and economic growth indexes. }\end{array}$ \\
\hline $\begin{array}{c}\text { Blocking effect } \\
\text { Self-sufficiency } \\
\text { syndrome effect }\end{array}$ & $\begin{array}{c}\text { Clustered firms often create entry barriers for new entrants. In the medium term, } \\
\text { it may turn some regional markets into oligopoly or even monopoly structures. } \\
\text { The reliance of clustered firms on their previous experience and successful } \\
\text { practices, applied in the past. In the medium term, it decreases region } \\
\text { innovation capacity. }\end{array}$ \\
\hline Lock-in effect & $\begin{array}{c}\text { Potential focus of clustered firms on local contracts, which often leads to a lack } \\
\text { of flexibility and, therefore, disability to adapt to external market changes }\end{array}$ \\
\hline
\end{tabular}

The negative effects of industrial clustering presented are among social and economic impacts that regional policymakers don't expect and cannot forecast. This situation is caused by insufficient theorizing on an industrial cluster and a region interaction. Table 1 shows, that most of the effects have a mixed nature (geographic and economic), whereas, mainstream approaches to industrial clustering consider an industrial cluster and its external interactions, focusing on one of two sides only: geographical (classical, agglomeration and administrative approaches) or economic (system, institutional and network approaches). Within this paper, to provide a complex view on industrial clustering negative effects genesis, we use the system and agglomeration approach, presented in our previous investigations [10]. Drawing from an equal focus on the geographic and economic sides of clustering, this approach considers clusters as spatial and economic structures, that are situated at the frontiers of a particular geographical area. In this respect, the system and agglomeration approach interprets genesis of industrial cluster effects on a region as a function, from 5 key factors of cluster internal milieu (fig 2). 


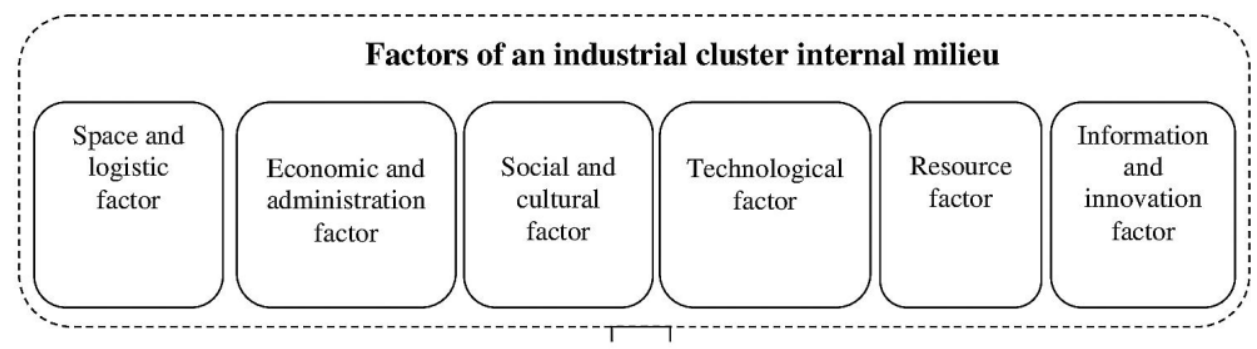

Industrial cluster influence on region social and economic growth

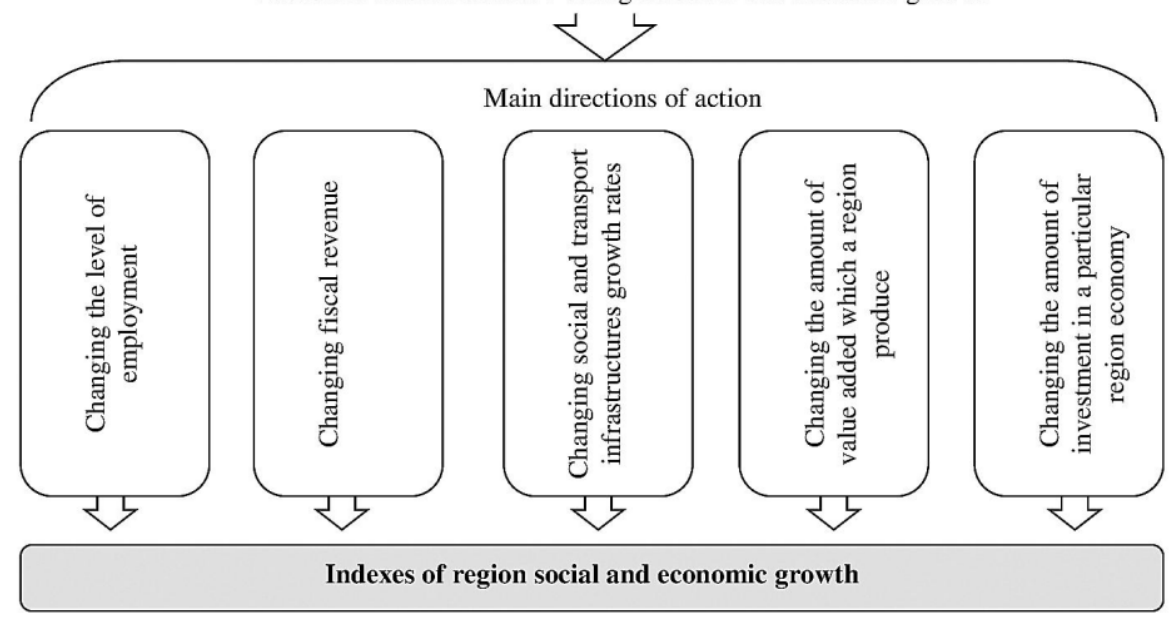

Fig. 2. Schematic diagram of the tailings dam topping by type

The space and logistic factor illustrates the logistic services and transport infrastructure that clustered firms commonly use. The factor mentioned stimulates industrial clusters to invest in regional transport infrastructure development, which often leads to a rise in regional employment level, Gross Regional Product (GRP), and, therefore, to Regional Living Standard (RLS) growth. Meanwhile an industrial cluster tends to invest mainly in the transport infrastructure of the geographic area where it is located. As a consequence, the area mentioned attracts new business residents. In some cases, it may result in an overconcentration effect [11].

The economic and administration factor reflects clustered firms strategic coordination, which provides them with relative economic sustainability and the ability to realize large common projects. The business activity mentioned attracts investments and increases the regional level of employment. Meanwhile, the aforementioned business coordination of clustered firms increases their common market power, which often causes blocking effects and, as a consequence, a downward tendency in the regional level of employment and GRP [3].

The social and cultural factor describes the associative culture shared by clustered firms, which allows them to reduce their transaction costs. In the medium term, the process mentioned stimulates GRP growth. However, the sustainable associative culture often causes self-sufficiency syndrome and lock-in effects [12].

The technological factor presents common technology that clustered firms use, forming a value-added chain. This technology results in clustered firms sustainability growth. Therefore, the quality of industrial clustered production rises. Nevertheless, the process 
mentioned develops "vulnerability to specialization effect", which, in some extreme cases, may cause regional economic slowdown $[12 ; 1]$.

The resource factor illustrates an industrial cluster ability to attract new suppliers in the region, which follows to growth in regional employment level and GRP. Meanwhile, the reliance of clustered firms causes Lock-in effects [3; 12].

The information and innovation factor illustrates the common information field that clustered organizations share. The field mentioned decreases transaction costs and attracts innovations to a region, stimulating the regional level of employment growth. However, clustered firms often close access to the field mentioned for new entrants. As a consequence, the regional start-ups survival rate decreases. Besides, it causes a selfsufficiency syndrome effect. In the medium term, these effects determine a decrease in region innovation capacity, decrease in amount of innovation in the region and, therefore, decrease in GRP [13].

\section{Conclusion}

To sum up within this investigation, we realised a complex and systematic conceptual analysis of the industrial clustering negative side. Drawing from the system and agglomeration approach, we illustrated the genesis of industrial clustering negative effects on a region. The proposed conceptual mechanism shows that most of the negative effects mentioned are connected with the tendency of each industrial cluster to close in on itself in the medium term. Hence, the key recommendation for regional policymakers to prevent negative effects of industrial clustering on regional social and economic growth is to develop industry-specific competition, via supporting innovation oriented firms. This simple measure is potentially able to decrease negative effects of industrial clustering and make regional economics more effective.

\section{References}

1. A. Azhar, The Pakistan Development Review, 4, 58 (2019)

2. F.C. Saadatyar, Strategic Change, 29, 227 (2020)

3. D. Fornahl, The life cycle of clusters: A policy perspective (2017)

4. M. Porter, Competitive Advantage of Nations (1990)

5. S. Brusco, Cambridge Journal of Economics, 58, 167 (1982)

6. A. Weber, Theory of the Location of Industries (1990)

7. O.E. Williamson, The mechanisms of governance (1996)

8. R.G. Giadadhubli, Indian J. of Regional Science, 9, 13 (1977)

9. S. Czamanski, Papers in Regional Science, 27, 137 (1971)

10. D.A. Koshcheev, E.A. Tretyakova, Russian J. of Economic Theory, 17, 17 (2020)

11. M. Fujita, The Japanese Economic Review, 54, 121 (2019)

12. N. Stojčić, Forest Policy and Economics, 109, 1 (2010)

13. T.F. Slaper, Economic Development Quarterly, 32(1), 44 (2018) 